\title{
Imaging acute thermal burns by photoacoustic microscopy
}

\author{
Hao F. Zhang \\ Konstantin Maslov \\ Texas A\&M University \\ Department of Biomedical Engineering \\ Optical Imaging Laboratory \\ 3120 TAMU \\ College Station, Texas 77843-3120
}

\section{George Stoica}

Texas A\&M University

Department of Veterinary Pathobiology

College Station, Texas 77843-5547

\author{
Lihong V. Wang \\ Texas A\&M University \\ Department of Biomedical Engineering \\ Optical Imaging Laboratory \\ 3120 TAMU \\ College Station, Texas 77843-3120
}

\begin{abstract}
The clinical significance of a burn depends on the percentage of total body involved and the depth of the burn. Hence a noninvasive method that is able to evaluate burn depth would be of great help in clinical evaluation. To this end, photoacoustic microscopy is used to determine the depth of acute thermal burns by imaging the total hemoglobin concentration in the blood that accumulates along the boundaries of injuries as a result of thermal damage to the vasculature. We induce acute thermal burns in vivo on pig skin with cautery. Photoacoustic images of the burns are acquired after skin excision. In a burn treated at $175^{\circ} \mathrm{C}$ for $20 \mathrm{~s}$, the maximum imaged burn depth is $1.73 \pm 0.07 \mathrm{~mm}$. In burns treated at $150^{\circ} \mathrm{C}$ for $5,10,20$, and $30 \mathrm{~s}$, respectively, the trend of increasing maximum burn depth with longer thermal exposure is demonstrated. (0 2006 Society of Photo-Optical Instrumentation Engineers. [DOI: 10.1117/1.2355667]
\end{abstract}

Keywords: noninvasive imaging; photoacoustic imaging; partial-thickness burn; thermal injury.

Paper 06126LR received May 18, 2006; revised manuscript received Jul. 17, 2006; accepted for publication Jul. 19, 2006; published online Sep. 21, 2006.
The extent and depth of burn injury are the two most important parameters for the clinical evaluation of burns. ${ }^{1}$ Burn wounds are categorized by depth into superficial, partialthickness, and full-thickness burns. With both very shallow (superficial, 0.07 to $0.12-\mathrm{mm}$ ) and very deep (full-thickness, $>2-\mathrm{mm}$ ) burns, clinical assessment of the burn depth is generally accurate. However, the most complicated cases are partial-thickness burns, which range from 0.12 to $2 \mathrm{~mm}$ and are further divided into superficial partial-thickness and deep partial-thickness burns. Owing to the destruction of the epidermis and a portion of the underlying dermis, clinical decisions about partial-thickness burns are difficult to make and often inaccurate. ${ }^{2,3}$ Because superficial partial-thickness burns heal well without surgical intervention while deep partialthickness burns require immediate medical attention or skin grafting, accurate determination of burn depth by noninvasive technologies is necessary.

Existing techniques that can measure burn depth noninvasively include laser Doppler perfusion imaging ${ }^{4}$ (LDPI), polarization-sensitive optical coherence tomography ${ }^{5,6}$ (PSOCT), indocyanine green dye fluorescence, thermography, ultrasound, and nuclear magnetic resonance imaging. Among these techniques, LDPI has drawn the most attention, and PSOCT is considered to have the most potential for the quantitative estimation of actual burn depth. ${ }^{2}$ LDPI detects the reduced blood flow that results from damaged dermal vasculature to estimate depth through a 2-D mapping of blood perfusion. However, because it uses a laser with a long coherence length, LDPI cannot provide depth resolved information, and

Address all correspondence to Lihong Wang, Biomedical Engineering, Washington University in St. Louis, One Brookings Drive, Campus Box 1097, St. Louis, MO 63130; Tel: 314-935-6152; Fax: 314-935-7448; E-mail: Ihwang@biomed.wustl.edu the correlation between the perfusion value and the burn depth is not straightforward. ${ }^{2}$ Moreover, LDPI is seriously affected by the optical heterogeneity of tissue. PSOCT measures the depth directly by acquiring cross-sectional images of thermal injuries with high spatial resolution. It images the optical polarization changes in tissue that result from the thermal destruction of collagen fibers. But because PSOCT detects only the direct-reflected (singly scattered) photons, its imaging depth in biological tissues is fundamentally limited to less than $1 \mathrm{~mm}$ due to strong optical scattering. This depth limit makes PSOCT unsuitable for imaging partial-thickness burns that are deeper than $1 \mathrm{~mm}$. Consequently, a new noninvasive technique that provides high-resolution depthresolved imaging of burns at a depth of a few millimeters offers great promise.

Here, we report on the imaging of thermal burn injuries using photoacoustic microscopy ${ }^{7,8}$ (PAM). Photoacoustic (PA) imaging is a hybrid volumetric imaging technology with high spatial resolution and good penetration depth. It images internal optical absorption distributions by detecting laser-induced photoacoustic waves (PA waves) with an ultrasonic detector. ${ }^{9}$

The resolution of PAM is determined by the focal diameter and the bandwidth of the ultrasonic detector. Using an ultrasonic transducer with a central frequency of $50 \mathrm{MHz}$, a bandwidth of $40 \mathrm{MHz}$, and a numerical aperture of 0.44 , PAM can reach an axial resolution of $15 \mu \mathrm{m}$ and a lateral resolution of $45 \mu \mathrm{m}$. Because a PA wave is generated by absorbed photons, PAM can image deeper than PSOCT by taking advantage of deeply-penetrating diffuse photons. At $50 \mathrm{MHz}$, the maximum imaging depth of PAM has been measured ${ }^{8}$ to be $3 \mathrm{~mm}$, which is limited primarily by strong frequency-dependent ultrasonic attenuation. The ratio of maximum imaging depth to

1083-3668/2006/11(5)/054033/5/\$22.00 @ 2006 SPIE 
axial resolution, which defines the number of effective pixels of PAM, is comparable to that of PSOCT and can achieve a value of more than 100. Hence, PAM is considered a highresolution imaging modality. Moreover, PAM possesses an additional advantage in that its imaging depth can be increased by reducing the ultrasonic frequency. Although this results in degraded spatial resolution, the depth-to-resolution ratio is preserved within certain ultrasonic frequency range. In contrast to LDPI, PAM provides high-resolution depthresolved imaging. In contrast to PSOCT, PAM images more deeply and can, therefore, cover the whole depth range of partial-thickness burns.

In biological tissues, oxyhemoglobin and deoxyhemoglobin are the dominating optical absorbers within a wide spectral range. Therefore, PAM is highly sensitive to blood. PAM measures burn depth based on imaging the hyperemia of acute thermal injuries, which have increased optical absorption compared with healthy and thermally coagulated tissues due to the higher blood content. ${ }^{10,11}$ The feasibility of using PA profiling to evaluate burn depth has been demonstrated in rats. However, only poor spatial resolution has been achieved. ${ }^{12,13}$

We used White Yorkshire pigs (20 to $30 \mathrm{~kg}$ ) with low skin melanin concentration as our animal model since pig skin has the closest anatomical resemblance to human skin. ${ }^{14}$ All experimental animal procedures were carried out in conformity with the guidelines of the National Institutes of Health ${ }^{15}$ (NIH). The laboratory animal protocol for this work was approved by the University Laboratory Animal Care Committee of Texas A\&M University.

After anesthetizing an animal with an intramuscular injection of xylazine at a dosage of $2.2 \mathrm{mg} / \mathrm{kg}$ and Telazol at a dosage of $5 \mathrm{mg} / \mathrm{kg}$, an area of $10 \times 10 \mathrm{~cm}^{2}$ on the pig back was depilated with commercial human hair remover lotion. An acute burn was created by applying electric cautery (Thermal Cautery Unit 150, Geiger Medical Technologies, Iowa; preheated to $175^{\circ} \mathrm{C}$ ) to the depilated skin area. After heating, edema and tissue coagulation were observed without obvious carbonization. A series of acute burns with different depths of damage were inflicted by varying the skin exposure to the cautery preheated to $150^{\circ} \mathrm{C}$. During each heating period, the pressure applied to the skin through the cautery was kept constant. The burned skin together with a large area of adjacent normal skin and subcutaneous tissues were immediately excised to a depth of $10 \mathrm{~mm}$ for the PAM imaging. After the skin excision, the animals were euthanatized by an intravenous administration of pentobarbital at a dosage of $100 \mathrm{mg} / \mathrm{kg}$. All of the burns were classified by histological results as deep partial-thickness burns.

The PAM system (Fig. 1) used a tunable dye laser (ND6000, Continuum, California) pumped by an Nd:YAG laser (Brilliant B, Bigsky, Montana; pulse duration: $6.5 \mathrm{~ns}$; repetition rate: $10 \mathrm{~Hz}$ ) as the radiation source. The laser light at the 584-nm wavelength was delivered through an optical fiber. At the 584-nm optical wavelength, oxyhemoglobin and deoxyhemoglobin have identical molar extinction coefficients. Therefore, the optical absorption is sensitive only to the total hemoglobin concentration rather than to the different forms of hemoglobin oxygenation. This optical wavelength agreed with the previously demonstrated optimal spectral range in PA burn profiling. ${ }^{13}$ The light coming out of the fiber was first ex-

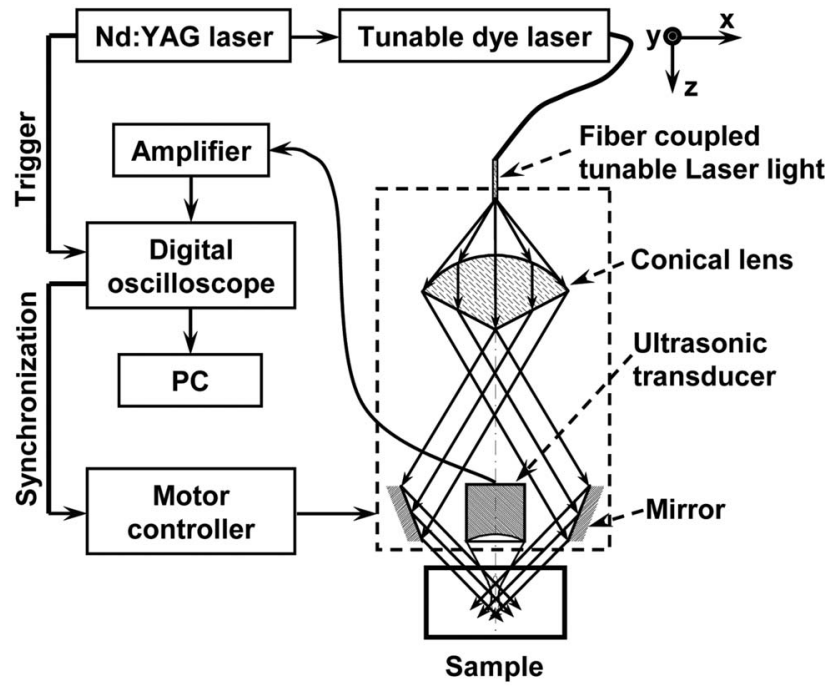

Fig. 1 Schematic of the PAM system.

panded by a conical lens and then weakly focused into samples by an optical condenser. An annular optical illumination was, therefore, formed on the sample surface with a dark center. The incident energy density on the sample surface, as well as around the optical focus, was controlled well within ANSI safety limits. ${ }^{16}$ A high-frequency ultrasonic transducer (V214-BB-RM, Panametrics, Washington) with a custommade spherical focusing lens was placed coaxially with the optical lenses. The focal region of the transducer and the optical focus overlapped each other to produce a confocal configuration. The PA signals received by the transducer were amplified by a wideband low-noise amplifier (ZFL-500LN, Mini-Circuits, New York) and were subsequently recorded by a digital oscilloscope (TDS 5034B, Tektronix, Oregon). In this study, 20 times signal averaging was applied to increase the SNR for acquiring cross-sectional images.

To acquire an image, PAM recorded the PA wave for $2 \mu \mathrm{s}$ at each location of the ultrasonic transducer. After being normalized by the corresponding laser pulse energy, the timeresolved PA signal was converted into a one-dimensional depth-resolved image (A-line) based on the sound velocity in soft tissue $(1.54 \mathrm{~mm} / \mu \mathrm{s})$ along the $z$ axis. Next, rasterscanning in the horizontal $(x-y)$ plane with a step size of $50 \mu \mathrm{m}$ produced a 3 -D image. Two types of 2-D images were used in this work: (1) cross-sectional (B-scan) images along the $z-x$ plane and (2) maximum-amplitude-projection (MAP) images along the $x-y$ plane, where the maximum PA amplitude of each A-line was projected along the $z$ axis.

Figure 2(a) shows a photograph, taken from the epidermal side after skin excision, of an acute skin burn that was heated at $175^{\circ} \mathrm{C}$ for $20 \mathrm{~s}$. A volume of $10 \times 10 \times 3 \mathrm{~mm}^{3}$ that covered the burn was imaged by PAM. Due to the optical and strong acoustic attenuations, the acquired PA signals from the hyperemic bowl on the skin surface had larger amplitudes than those from below. Therefore, the MAP image [Fig. 2(b)] shows only the hyperemic ring on the skin surface. For B-scan PAM images, amplitude compensation along the $z$ axis was applied to better visualize the deeper structures. Since acoustic attenuation is the dominating factor at the $50-\mathrm{MHz}$ 


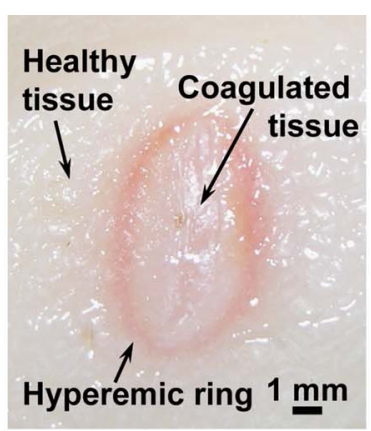

(a)

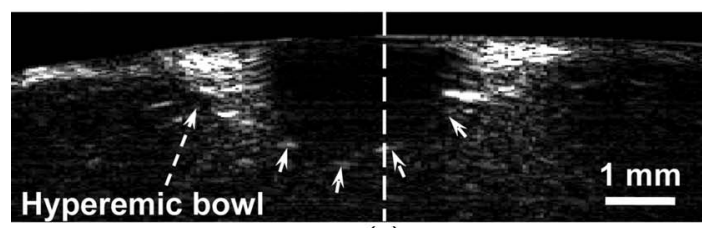

(c)

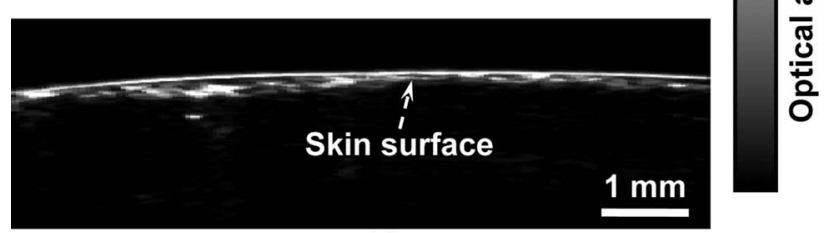

(d)

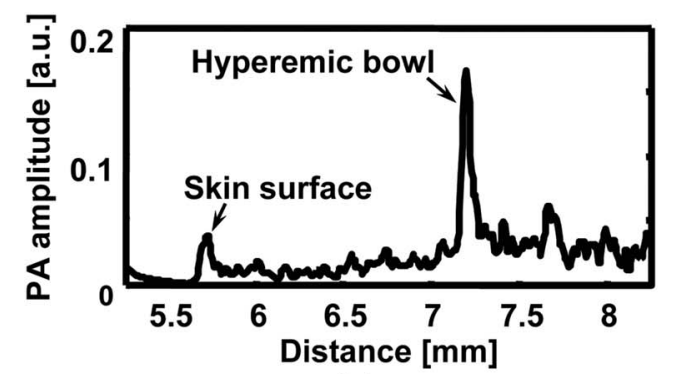

(e)

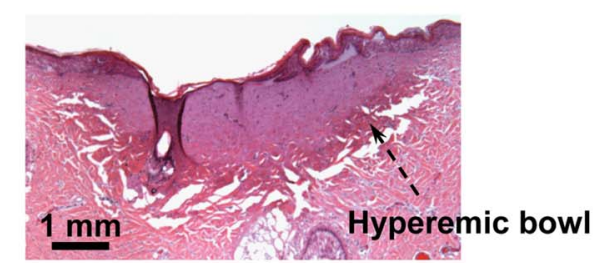

(f)

Fig. 2 PAM image of an acute skin burn heated at $175^{\circ} \mathrm{C}$ for $20 \mathrm{~s}$ : (a) photograph of the burn from the epidermal side, (b) MAP image showing the morphology of the hyperemic ring, (c) B-scan image of the hyperemic bowl at the location marked in panel (b), (d) typical B-scan image of healthy skin, (e) A-line at the location marked in panel (c), and (f) hematoxylin-and-eosin (HE) stained histological section.

central frequency, the measured PA amplitudes at difference depths beneath the skin surface were compensated for based on the acoustic attenuation in porcine skin (2.5 $\mathrm{dB} \mathrm{cm}^{-1} \mathrm{MHz}^{-1}$; Ref. 17). This compensation provided a good first-order estimation of the true PA amplitudes generated from deep structures for morphological imaging purposes. The compensated B-scan image of the burn at the marked location in Fig. 2(b) is given in Fig. 2(c).

A typical acute thermal injury consists of three distinct regions: (1) a central zone of edema and coagulated tissue, where no blood flow is expected; (2) a red band called the hyperemic bowl (hyperemic ring when on the skin surface,) which results from hemorrhaging at the periphery of the coagulated zone; and (3) an outside thermally intact healthy tissue area. ${ }^{18}$ The hyperemic bowl represents the area in which hemostasis, hemorrhage, and hyperemia occur as a result of thermal damage to the blood vessels and the pathophysiologic response to heating. Hence it defines the boundary of the thermal damage. These three zones are clearly observed in Fig. 2(a). Because PAM is an optical absorption-based imaging modality and hemoglobin is the dominant optical absorbing pigment at the 584-nm wavelength, the contrast in the PAM imaging of acute burns comes from the differences in hemoglobin concentration. Although optical scattering varies prominently in coagulated tissues compared with that in healthy tissues, only a small change was found in the optical absorption around the 584-nm wavelength. ${ }^{10}$ For edema, both optical scattering and absorption decrease compared with normal tissue. ${ }^{19}$ As a result, the hyperemic bowl generates a higher PA amplitude due to the higher hemoglobin concentration than do the healthy and thermally coagulated tissues. In Fig. 2(b), the ratio of the imaged PA amplitude of the hyperemic ring to that of the inner coagulated tissue was $20.4 \pm 3.4$; and the ratio of the imaged PA amplitude of the hyperemic ring to that of the outer normal tissue was $9.5 \pm 1.9$. Comparison with the photograph [Fig. 2(a)] shows that both the morphology and the size of the hyperemic ring were correctly imaged by PAM.

In Fig. 2(c), the middle dark region represents the thermally coagulated tissue, whose boundary is defined by the brighter hyperemic bowl as marked by the arrows. Because capillaries are beyond the spatial resolution of the current PAM system, no obvious structures were imaged in the outside healthy tissue in this burn sample. A typical B-scan of normal healthy skin is shown in Fig. 2(d) for comparison after the same compensation for acoustic attenuation.

Once the B-scan image of the hyperemic bowl was acquired, the burn depth was estimated based on the distance between the imaged skin surface and the inner boundary of the hyperemic bowl. Figure 2(e) gives the A-line at the location marked in Fig. 2(c), where the horizontal axis represents the distance from the ultrasonic transducer. The two PA peaks in the A-line correspond to the skin surface and the inner boundary of the hyperemic bowl, respectively. Thus, the distance between these two peaks was taken as an estimate of the burn depth. After a lateral neighborhood averaging within a region of $0.5 \mathrm{~mm}$ along the $x$ axis in Fig. 2(c), the maximum burn depth was measured to be $1.73 \pm 0.07 \mathrm{~mm}$. A histological result showing the cross section of a hyperemic bowl is shown in Fig. 2(f).

Figures 3(a)-3(d) show the B-scan images of the acute skin burns that were heated at $150^{\circ} \mathrm{C}$ for $5,10,20$, and $30 \mathrm{~s}$, respectively. Based on the imaged inner boundaries of the hyperemic bowls, increased burn depth was observed for the longer thermal exposures, which agrees with the pathogenesis of acute burns. ${ }^{11}$ Moreover, the imaged width of the hyperemic bowls on the skin surface also increased with longer heating time, which was supported by direct observation be- 


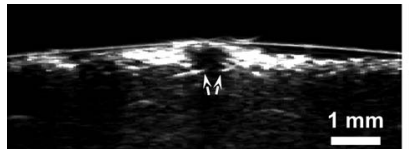

(a)

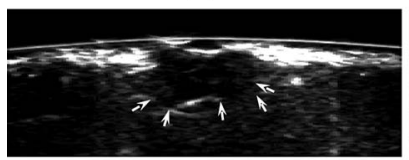

(c)

(e)



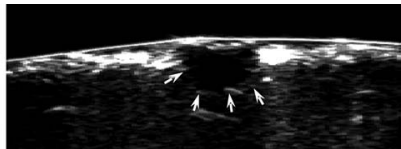

(b)

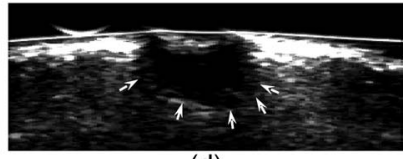

(d)



(f)
Fig. 3 PAM images of acute skin burns with different depths introduced by changing the heating durations to (a) 5, (b) 10, (c) 20, and (d) $30 \mathrm{~s}$. (e) Photograph of the cross section shown in panel (d), and (f) imaged change of the maximum burn depth with heating duration.

fore the skin excisions. The photograph in Fig. 3(e) shows the same cross section as shown in Fig. 3(d) of the 30-s burn. Both the imaged width and depth of the hyperemic bowl fit the photograph. The measured maximum burn depth versus the heating duration is given in Fig. 3(f), where the trend associated with increasing burn depth is further demonstrated.

Compared to LDPI and PSOCT, which must correlate the measured parameters, such as blood perfusion and polarization changes, to the actual burn depth, PAM provides a direct assessment of the true boundaries of the burns by imaging the hyperemic bowl. Because vascular damage is a much more sensitive indicator than parenchymal change, e.g., denatured collagen, ${ }^{3}$ PAM has the potential to achieve high accuracy in assessing burn depth. Moreover, PAM is not affected by edema because edema introduces only small optical absorption changes relative to the strong optical absorption of hemoglobin. Some exceptions may exist in postacute burns and slow burns, where the boundaries of the thermal damage may not be well defined by the hyperemic bowl. ${ }^{5}$ Since the imaged burn depth measures up to $1.73 \pm 0.07 \mathrm{~mm}$, PAM is well suited for imaging human skin.

Compared with the previous PA results, the results shown here achieved a sixfold improvement in axial resolution and a 40-fold improvement in lateral resolution, which enables much higher precision in measuring burn depths. Moreover, porcine skin is more similar to human skin than rat skin; thus, this study represents progress toward human applications.

In this study, due to the lack of flexibility of the desktop PAM system, the acute burns were imaged immediately after skin excision, and a single optical wavelength at the isosbestic point was used to demonstrate the feasibility. However, PAM itself is a noninvasive technology and a handheld version of PAM is under development, which will be operated similarly to a traditional ultrasound imager to enable us to perform in vivo studies. To better understand the pathophysiology of burn injuries, hemoglobin oxygen saturation will be measured using multiwavelength PAM imaging. ${ }^{8}$ A time course study of the change in hemoglobin oxygen saturation will also be con- ducted to investigate postburn reactions and to examine healing. ${ }^{20}$

There are two technical limitations to the current PAM. First, because of the low laser repetition rate $(10 \mathrm{~Hz})$, data acquisition for a B-scan image (20 times averaging) takes 10 min. A new system with a laser repetition rate of up to $1 \mathrm{kHz}$ is under construction, which will significantly reduce the data acquisition time. The second limitation is that PAM requires a coupling medium (Ultrasound scanning gel, Sonotech, Washington) between the ultrasonic detector and the damaged tissues in order to detect the induced PA waves. This contact may possibly create difficulties in clinical environments. However, no mechanical pressure was applied through the ultrasound gel, and commercially available sterilized ultrasound gel could be used to prevent infections.

In summary, the feasibility of imaging acute burns and thus determining their depths using PAM was demonstrated by imaging the hyperemic bowl. High imaging contrast and resolution were achieved in three dimensions based on the differences in total hemoglobin concentration and strong ultrasonic focusing. Burn depths of up to $1.73 \pm 0.07 \mathrm{~mm}$ were imaged, and increased burn depth related to longer heating period has been observed. In addition to structural imaging, functional imaging is possible by performing spectral measurements in vivo.

\section{Acknowledgments}

We thank Dr. Geng Ku and Xueyi Xie for experimental assistance. This project is sponsored by National Institutes of Health grants R01 EB000712 and R01 NS46214.

\section{References}

1. D. Heimbach, L. Engrav, B. Grube, and J. Marvin, "Burn depth: a review," World J. Surg. 16, 10-15 (1992).

2. B. S. Atiyeh, S. W. Gunn, and S. N. Hayek, "State of the art in burn treatment," World J. Surg. 29, 131-148 (2005).

3. A. Watts, M. Tyler, M. Perry, A. Roberts, and D. McGrouther, "Burn depth and its histological measurement," Burns 27, 154-160 (2001).

4. D. Park, J. Hwang, K. Jang, D. Han, K. Ahn, and B. Baik, "Use of laser Doppler flowmetry for estimation of the depth of burns," Plast. Reconstr. Surg. 101, 1516-1523 (1998).

5. S. Srinivas, J. de Boer, H. Park, K. Keikhanzadeh, H. Huang, J. Zhang, W. Jung, Z. Chen, and J. Nelson, "Determination of burn depth by polarization-sensitive optical coherence tomography," $J$. Biomed. Opt. 9, 207-212 (2004).

6. S. Jiao, W. Yu, G. Stoica, and L. V. Wang, "Phase-based polarization contrast Mueller optical coherence tomography and application in burn imaging," Appl. Opt. 42, 5191-5197 (2003).

7. K. Maslov, G. Stoica, and L. V. Wang, "In vivo dark-field reflectionmode photoacoustic microscopy," Opt. Lett. 30, 625-627 (2005).

8. H. F. Zhang, K. Maslov, G. Stoica, and L. V. Wang, "Functional photoacoustic microscopy for high-resolution and noninvasive in vivo imaging," Nat. Biotechnol. 24, 848-851 (2006).

9. X. Wang, Y. Pang, G. Ku, X. Xie, G. Stoica, and L. V. Wang, "Noninvasive laser-induced photoacoustic tomography for structural and functional imaging of the brain in vivo," Nat. Biotechnol. 21, 803 806 (2003).

10. S. Thomsen, H. Vijverberg, R. Huang, and J. Schwartz, "Changes in optical properties of rat skin during thermal coagulation," in Proc. SPIE 1882, 230-236 (1993).

11. A. R. Moritz, "Studies of thermal injury III. The pathology and pathogenesis of cutaneous burns an experimental study," Am. J. Pathol. 23, 915-941 (1947).

12. S. Sato, M. Yamazaki, D. Saitoh, H. Tsuda, Y. Okada, M. Obara, and H. Ashida, "Photoacoustic diagnosis of burns in rats," J. Trauma: Inj., Infect., Crit. Care 59, 1450-1455 (2005). 
13. M. Yamazaki, S. Sato, H. Ashida, D. Saito, Y. Okada, and M. Obara, "Measurement of burn depth in rats using multiwavelength photoacoustic depth profiling," J. Biomed. Opt. 10, 064011-1-4 (2005).

14. A. R. Moritz and F. C. Henriques, "Studies of thermal injury II. The relative importance of time and surface temperature in the causation of cutaneous burns," Am. J. Pathol. 23, 695-720 (1947).

15. National Institutes of Health, "Guide for the care and use of laboratory animals," NIH Pub. 86-23, U.S. Government Printing Office, Washington, DC (1985).

16. American National Standards Institute, "American national standard for the safe use of lasers," ANSI Standard Z136.1-2000, ANSI, Inc., New York (2000).

17. T. Baldeweck, P. Laugier, and G. Berger, "An in vitro study on por- cine skin: attenuation profile estimation using auto-regressive modeling," in Proc. IEEE Ultrasonic Symp., Vol. 2, pp. 1141-1144 (1995).

18. B.-M. Kim, S. L. Jacques, S. Rastegar, S. Thomsen, and M. Motamedi, "Nonlinear finite-element analysis of the role of dynamic changes in blood perfusion and optical properties in laser coagulation of tissue," IEEE J. Sel. Top. Quantum Electron. 2, 922-933 (1996).

19. Y. Ueda, S. Sato, H. Ashida, H. Ooigawa, H. Nawashiro, K. Shima, D. Saitoh, Y. Okada, and M. Obara, "Transcranial measurement of diffuse light reflectance from cold-injured brains in rats," J. Biomed. Opt. 10, 064010-1-7 (2005)

20. A. Tandara and T. Mustoe, "Oxygen in wound healing - more than a nutrient," World J. Surg. 28, 294-300 (2004). 